\title{
A Novel 2.45GHz Compact Wideband Micro strip Antenna
}

\author{
Hanchun Chen ${ }^{1, a, ~}{ }^{*}$, Xinglong Guo, b \\ ${ }^{1}$ School of electronic information, Nantong University, Nantong 226019, China. \\ ${ }^{2}$ School of electronic information, Nantong University, Nantong 226019, China. \\ a758191768@qq.com, bxlguo@ntu.edu.cn
}

Keywords: micro strip patch antenna, miniaturization, Increase antenna gain.

\begin{abstract}
In order to solve the contradiction of bandwidth narrowing and gain reduction caused by miniaturization of micro strip patch antenna,this paper designs a novel miniaturized wideband micro strip antenna by combining slotting on radiation patch with slotting on ground plate. The miniaturization of the antenna is achieved by opening the double T-slots on the patch and optimized by opening the double U-grooves on the ground plate, not only significantly widening the bandwidth of the antenna, but also effectively increasing the gain of the antenna. The simulation results show that the antenna size is reduced by $48.83 \%$ compared with the original theoretical value at the center frequency of $2.45 \mathrm{GHz}$. Compared with the pre-optimization, the relative bandwidth of the optimized antenna is extended from $1.63 \%$ to $9.39 \%$, and the gain is increased from $-1.08 \mathrm{~dB}$ to $2.47 \mathrm{~dB}$, to achieve a good overall performance. The antenna has the advantages of low profile, simple structure, easy fabrication, low cost and good application prospect.
\end{abstract}

\section{Introduction}

Micro strip antennas are widely used in various wireless communication systems because of their advantages such as simple structure, small size, light weight, low profile and easy integration. However, micro strip antennas also have some inherent drawbacks. On the one hand, since the size of the micro strip antenna is inversely proportional to the frequency [1], when it works in the lower frequency range below $2.5 \mathrm{GHz}$, the size is large, and it cannot meet the requirements of mobile communication system toward miniaturization and integration. On the other hand, micro strip antenna is a kind of resonant antenna, its $Q$ value is so high that the bandwidth is very narrow. The bandwidth of general micro strip antenna is only $0.5 \% \sim 3.0 \%$, which severely limits its application. Therefore, miniaturization and broadband become the inevitable trend of the development of micro strip antennas.

At present, the technology of micro strip antennas miniaturization mainly include: using dielectric substrate with high dielectric constant, loading short circuit probe, grooving on the radiation patch and so on [2]. In [3], the micro strip antenna size is reduced by $75 \%$ with the method of high dielectric constant substrate; in [4], the size of the micro strip antenna is reduced by about $50 \%$ by loading the short-circuit probe; in [2], the size of the micro strip antenna is reduced by $20 \%$ by grooving on the radiation patch. However, the shortcomings of the above methods are that the antenna work bandwidth is narrow and the gain is low. Therefore, how to expand the bandwidth and ensure the antenna has enough gain is the problem that must be considered in the miniaturization design of micro strip antennas.

Although micro strip antennas have many ways to expand bandwidth and increase the gain, these methods tend to sacrifice the performance of other aspects of antennas. Such as damaging the low profile of the antenna, increasing the size of the antenna, complicating the structure of an antenna, etc. After consulting the recent papers on micro strip antennas, it is found that the bandwidth of the antenna can be extended greatly $[5,6,7,8]$ or the antenna gain can be improved to a certain extent by slotting in the proper position of the ground board $[2,9,10]$, but rarely found examples that can simultaneously expand bandwidth and enhance gain. On the basis of the above research, in consideration of the easy realization of slotting technology, this paper adopts the combination method 
of slotting on the radiation patch and grooving on the ground plate. The size of the antenna is reduced by opening the double T-slots on the radiation patch; by opening the double U-grooves in the proper position of the ground plate, not only the bandwidth of the antenna is greatly expanded, but also the gain is effectively increased, which effectively solves the drawback of bandwidth narrowing and gain reduction caused by miniaturization of micro strip antenna.

\section{Antenna Design Theory}

Typically, micro strip antennas consist of radiation patches, dielectric substrates, and ground plates. Because the design and manufacturing process of rectangular micro strip antenna are simple, rectangular design is adopted in this paper. According to the theory of micro strip antenna, the size calculation formula of rectangular patch antenna is as follows [1]:

$$
\begin{aligned}
& W=\frac{c}{2 f_{0}}\left(\frac{\varepsilon_{r}+1}{2}\right)^{-\frac{1}{2}} \\
& L=\frac{c}{2 f_{0} \sqrt{\varepsilon_{s}}}-2 \Delta L \\
& \varepsilon_{\varepsilon}=\frac{\varepsilon_{r}+1}{2}+\frac{\varepsilon_{r}-1}{2}\left(1+12 \frac{\hbar}{W}\right)^{-\frac{1}{2}} \\
& \Delta L=0.412 h \frac{\left(\varepsilon_{\mathrm{g}}+0 . \mathrm{a}\right)(W / \mathrm{h}+0.264)}{\left(\varepsilon_{\mathrm{q}}-0.25 \mathrm{~g}\right)(w / h+0.8)} \\
& \lambda_{g}=\frac{e}{f_{0} \sqrt{\varepsilon_{g}}} \\
& L G \geq L+0.2 \lambda_{g}, W G \geq W+0.2 \lambda_{g}
\end{aligned}
$$

Among them, $c$ is the speed of light, $f_{0}$ is the resonant frequency, $W$ and $L$ are respectively the width and length of the radiation patch, $\varepsilon_{r}$ is the relative dielectric constant, $\varepsilon_{\theta}$ is the effective dielectric constant, $\Delta L$ is the slot length of the equivalent radiation, $h$ is the thickness of the dielectric substrate, is the wavelength in the medium, $L G$ and $W G$ are respectively the length and width of the ground and dielectric substrate. When the size of the ground is satisfied (6), the ground can be regarded as infinitely large.

After comprehensive consideration, the low cost FR4 is chosen as the dielectric substrate, whose dielectric constant $\varepsilon_{\gamma}$ is 4.4 , and its thickness $h$ is set as $1.6 \mathrm{~mm}$. The center frequency of the antenna $f_{0}$ is $2.45 \mathrm{GHz}$, and the feeding mode is coaxial feeding. The known parameter values are substituted into the formula (1) to (4), and $W=37.26 \mathrm{~mm}, L=30.21 \mathrm{~mm}$ are obtained, and the size is slightly larger, so that the miniaturization design is needed.

\section{Compact Antenna Design}

Because the method of realizing the miniaturization of the antenna by the surface grooving is easy to implement, in this paper, the size of the antenna is reduced by opening symmetrical double T-slots on the non-radiation edges of the patch. The antenna model is shown in FIGURE 1, in which the center of the rectangular patch is coincident with the center of the ground plate. Since the antenna designed in this paper is a square antenna, there are $L=W, L G=W G$.

By opening the double T-slots on the patch, the current path of the patch surface is extended, thereby reducing the resonant frequency of the antenna. For the same operating frequency, the size of the antenna is reduced. The above antenna is simulated by a soft HFSS simulation software, and the size of the patch and T-slot and the position of feed point are optimized. In the simulation, we find that the main parameter that affects the resonant frequency of the micro strip antenna is the length of the slot, the longer the slot, the lower the resonant frequency of the antenna, while the width of the slot has no obvious influence on the resonant frequency. After several simulation and optimization, the final parameters of the antenna are: $L=24 \mathrm{~mm}, L G=36.68 \mathrm{~mm}$, $L_{1}=15.5 \mathrm{~mm}, W_{1}=1 \mathrm{~mm}, L_{2}=1.5 \mathrm{~mm}, W_{2}=1 \mathrm{~mm}, d_{1}=7.99 \mathrm{~mm}$. The simulation results are shown in Figure 2 and Figure 3. 


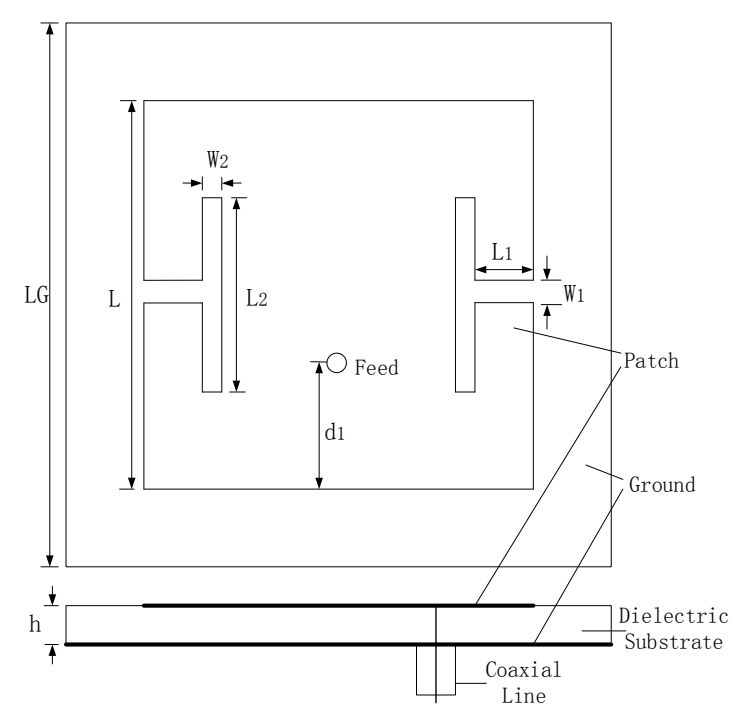

Figure 1. Micro Strip antenna

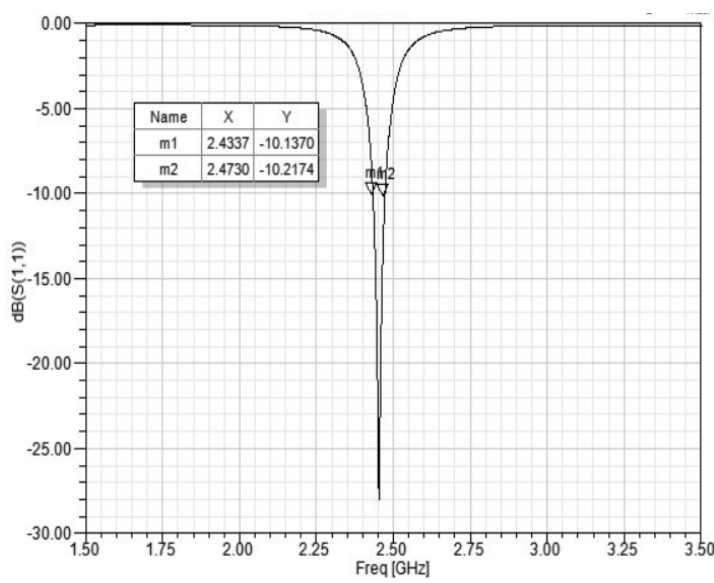

Figure 2. Simulation Result of Return Model Loaded T-Slots.

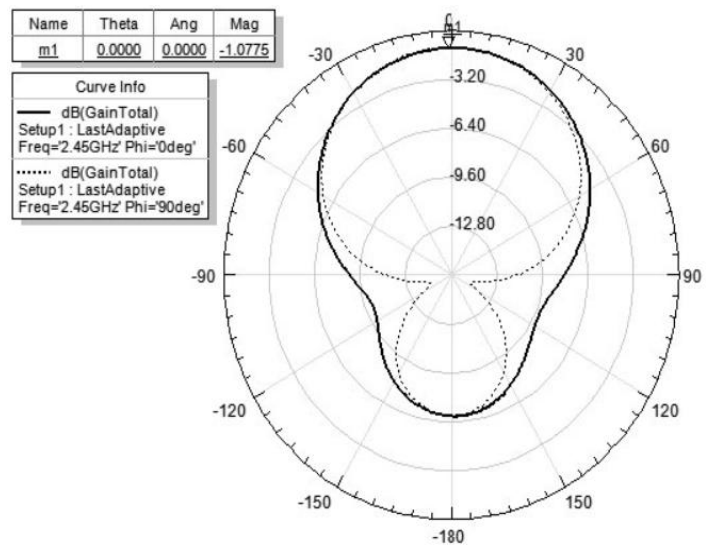

Figure 3. Simulation Result of Radiation Pattern of the Antenna

It can be seen from Figure 2 that the return loss $S_{11}$ of the antenna at $2.45 \mathrm{GHz}$ is about $-28 \mathrm{~dB}$, which shows that the antenna achieves a good matching performance at $2.45 \mathrm{GHz}$. At this point, the size of the antenna from the theoretical value of $30.21 \mathrm{~mm} \times 37.26 \mathrm{~mm}$ successfully reduced to $24 \mathrm{~mm}$ $\times 24 \mathrm{~mm}$, reduced by $48.83 \%$. But also to see that the $S_{11} \leq-10 \mathrm{~dB}$ bandwidth coverage of antenna is only $2.43 \mathrm{GHz} \sim 2.47 \mathrm{GHz}$, the absolute bandwidth is only about $40 \mathrm{MHz}$, and the relative bandwidth is only $1.63 \%$, it is difficult to meet the actual needs of broadband. The fundamental reason for the narrow bandwidth is that the $Q$ value of the micro strip antenna is too high, and it is difficult to achieve wideband by slotting the patch. In addition, the reduction of the overall size of the antenna also results in a reduction in bandwidth [11].

As can be seen from FIGURE 3, the maximum radiation gain of the antenna is $-1.08 \mathrm{~dB}$, which appears to be relatively low. Because of the reduction of the patch size and the slot on the patch, the radiation resistance and efficiency of the antenna are reduced, resulting in a decrease in the gain.

\section{Improvement of Antenna Structure}

Document [5] adopts the method of etching dumbbell slots on the ground plate below the two radiation edges, which greatly increases the bandwidth of the antenna without increasing the antenna volume. Inspired by this, on the basis of the antenna model shown in FIGURE 1, the bandwidth of the antenna is successfully achieved by double U-slots on the ground plate under the two radiation edges. Unlike the document [5], the improved antenna also increases the gain, and the model is shown in FIGURE 4. The improved antenna is simulated by using a soft HFSS simulation software, and the size 
of U-slot and the position of feeding point are optimized. After many optimizations and adjustments, the dimensions of the U-slot are $L_{a}=4 \mathrm{~mm}, W_{a}=2.5 \mathrm{~mm}, L_{4}=22 \mathrm{~mm}, W_{4}=0.5 \mathrm{~mm}$, the distance from the U-slot to the lower edge of the ground plate is $d_{2}=3.84 \mathrm{~mm}$, and the distance from the feed point to the lower edge of the patch is adjusted to $d_{1}=2.97 \mathrm{~mm}$. The simulation results of the improved antenna are shown in FIGURE 5 and FIGURE 6.

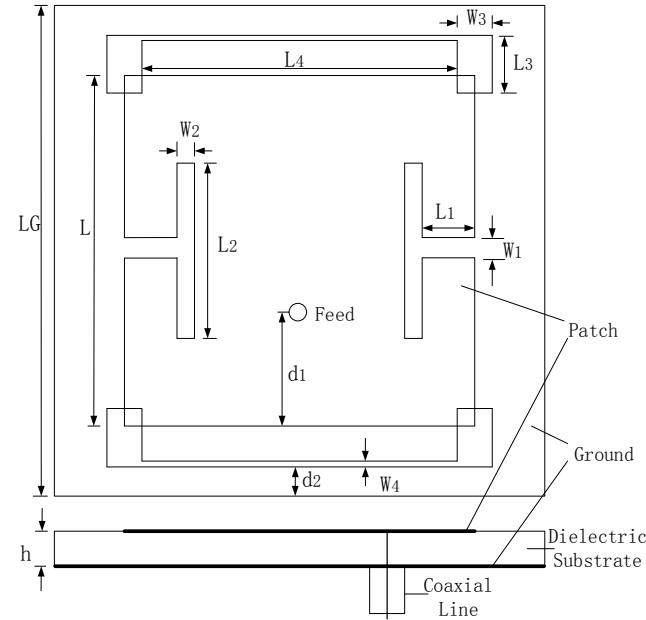

Figure 4. Micro Strip Antenna Model Loaded T-Slots.

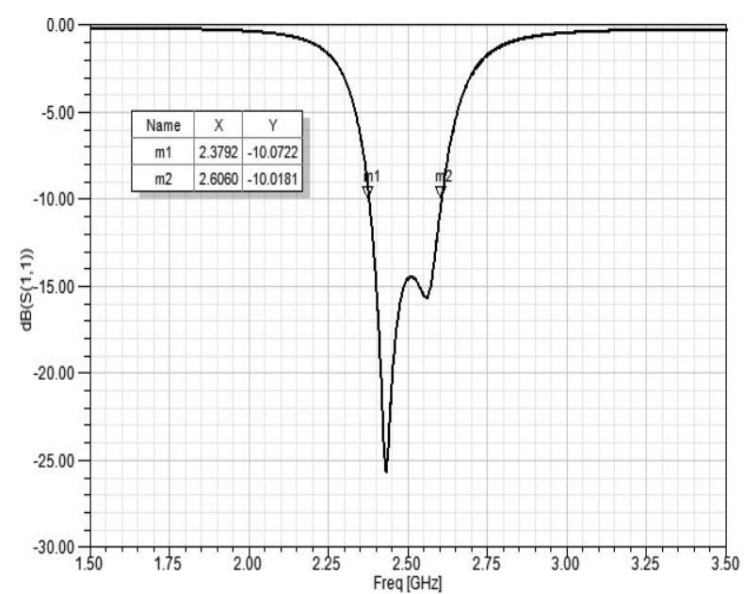

Figure 5. Simulation Result of Return Loss of the Antenna

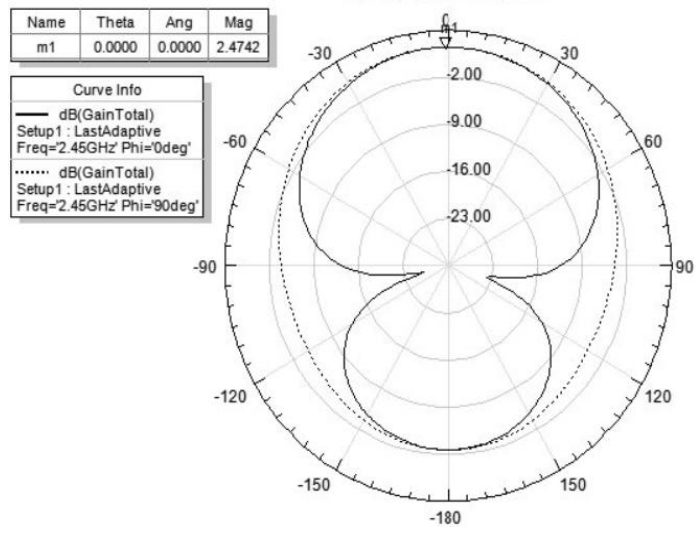

Figure 6. Simulation result of radiation pattern of the antenna

It can be seen from FIGURE 5, the $S_{11} \leq-10 \mathrm{~dB}$ bandwidth coverage of antenna extended to $2.38 \mathrm{GHz} \sim 2.61 \mathrm{GHz}$, the absolute bandwidth is about $230 \mathrm{MHz}$, and the relative bandwidth is $9.39 \%$, which is 5.75 times the bandwidth shown in FIGURE 2. This bandwidth completely covers the band of the WLAN $802.11 \mathrm{~b}(2.4 \sim 2.4835 \mathrm{GHz})$, thus meeting the needs of broadband. The slot on the ground of the micro strip antenna leads to the reverse leakage of some electromagnetic energy, thereby reducing the energy radiation quality factor $Q_{r}$ of the antenna [9]. Since the total quality factor of the antenna $Q \approx Q_{r}[11]$, it leads to the decrease of $Q$.Because the bandwidth of the antenna $B W=(V S W R-1) /(Q / \sqrt{V S W R})[5]$, where VSWR is the standing wave coefficient, the bandwidth $B W$ increases when the VSWR is constant and the $Q$ is down.

Can be seen from FIGURE 6, the maximum gain of the antenna radiation reached $2.47 \mathrm{~dB}$, which is $3.55 \mathrm{~dB}$ higher than the gain of $-1.08 \mathrm{~dB}$ shown in FIGURE 3, to meet the power demand of general micro strip antenna. According to the literature [1], the gain of the antenna is $\mathrm{G}=\eta_{A} \mathrm{D}$ in which $\eta_{A}$ is the efficiency of the antenna, $D$ is the directivity coefficient. According to the literature [11], when the antenna works in the main mode $\left(\mathrm{TM}_{10}\right)$, there is $D=\left(2 a^{2}\right) /\left(15 \lambda_{0}{ }^{2} Q_{r}\right)$, in which $a$ is the size of the antenna radiation edge, $\lambda_{\sigma}$ is the working wavelength, and $Q_{r}$ is the energy radiation quality factor. Thus, when $a, \lambda_{0}$ and $\eta_{A}$ are constant and $Q_{r}$ decreases, the gain increases.

To sum up, slotting at the proper position of the ground plate can not only obviously broaden the 
bandwidth of the antenna without increasing the volume of the antenna, but also greatly increase the antenna gain. Thus, the contradiction of bandwidth narrowing and gain reduction caused by miniaturization of antennas is effectively solved.

\section{Conclusion}

In this paper, the combination method of slotting on the radiation patch and grooving on the ground plate is adopted, which not only achieves the miniaturization of the antenna, but also broadens the bandwidth and maintains a good gain, thus achieving a good comprehensive performance. In addition, the antenna has the advantages of low profile, simple structure, easy fabrication, low cost and good application prospect.

\section{References}

[1]. Ming yang Li, Min Liu. HFSS Antenna Design (2nd Edition) [M]. Beijing: Electronic Industry Press, 2014.

[2]. Hong Yang, Li Guo. Design of a UHF band RFID handheld reader antenna [J]. Electronic Components and Materials, 2014, 33 (5): 71-74.

[3]. Hang ying, Yuan, Jieqiu Zhang. A miniaturized micro strip patch antenna for "Beidou" navigation system [J]. Journal of Air Force Engineering University (Natural Science Edition), 2012, 13 (5): 76-79.

[4]. Ning Hou, Tianyu Zhang. Simulation design of short-circuit pinned micro strip patch antenna based on HFSS [J]. Journal of Jishou University (Natural Science Edition), 2008, 29 (4): 53-57.

[5]. Gengqi Zheng, Baohua Sun. Study of a new broadband miniaturized micro strip antenna [J]. Journal of Xi'an Electronic and Science University (Natural Science Edition), 2017, 44 (4): $55-58$.

[6]. K.Yin, J. P. Xu. Design of a four-element multiple-input-multiple-output antenna for compact longterm evolution small-cell base stations [J]. Electronics Letter, 2008, 44 (7) : 453-454.

[7]. Boran Guan, Jianwei Cao. A small ultra-wideband micro strip antenna [J]. Journal of Microwave, 2011, 27 (2): 60-62, 92.

[8]. BBQ. Elias, H.M.AL-Dahhan. Bandwidth Enhancement of A micro strip Patch Antenna for C-band and X-band By Using New Structure of Defected Ground Technique [J].International Journal of Computer and Mobile Computing, 2015, 4 (1) : 426-432.

[9]. Xi Deng, Yunlin Liu, Zhiwei Shi, etc .A new type of ultra-miniaturized antenna [J]. Communication Technology, 2010, 43 (7) : 77-79.

[10]. R.Pandeeswari, S. Raghavan. Micro strip Antenna with Complementary Split Ring Resonator Loaded Ground Plane for Gain Enhancement [J].Microwave And Optical Technology Letters, 2015, 57 (2) : 292-296.

[11]. Gang Wen .UHF band new wide-band circular polarization conformal antenna [D]. University of Electronic Science and Technology, 2013. 